\title{
Considerations of Acupuncture Protocol for Treating Heart Blood Deficiency-Patterned Heart Diseases
}

\author{
Tong Zheng Hong* \\ As-You-Wish Healthcare Institute, Taiwan
}

Received: 阱 October 22, 2018; Published: 制 October 25, 2018

*Corresponding author: Tong Zheng Hong, As-You-Wish Healthcare Institute, MS in Acupuncture awarded by National University of Health Sciences in IL, Taiwan

\begin{abstract}
Most patients are not aware of the heart disease for years until a "sudden and fatal" heart attack resulted from coronary artery disease. Yin-Yang balance, the foundation and the guideline to explain that etiology of diseases that are understood as a loss of balance between Yin and Yang, which is the unique concept distinguishing TCM from Western medicine. ST36 and LV3 representing Qi and Blood are the essential acupoints to achieve the goal of Yin-Yang balance for Blood deficiency-patterned heart diseases.
\end{abstract}

Keywords: Yin-Yang; Qi and Blood; Blood deficiency; Ischemic heart disease

\section{Introduction}

The most common and easiest access of protein and fat humans consume is meat protein. It has been confirmed that protein plays a key role in the formation of atherosclerosis, a leading cause of death closely associated with increasing risk of heart disease $[1,2]$. Lipoprotein (a), for example, is a lipoprotein whose plasma concentration contributes to the risk of cardiovascular disease (CVD) and has been believed to be a risk factor for CVD in adults, independent of age, diet, physical activity, smoking history, ethanol consumption, and gender [3]. The coronary arteries bring blood and oxygen to the heart. Most of the patients with coronary artery disease are not aware of the heart disease for years until a "sudden and fatal" heart attack. Most of the symptoms and signs of coronary artery disease are often noted in the advanced state of disease as the disease progresses "silently" before the first onset of symptoms. Coronary artery disease (CAD) also known as atherosclerotic heart disease, coronary heart disease, or ischemic heart disease (IHD) is the most common type of heart disease that can cause heart attacks. In addition to symptoms of stable ischemic heart disease that include angina (chest pain) and decreased exercise tolerance. The risk of artery narrowing increases with age, smoking, high blood cholesterol, high blood pressure, and diabetes. Moreover, this artery issue is more common in men and those who have close relatives with CAD [3]. Quality of life of the senior can be extremely impaired by unexplained repetitively occurring Blood deficiencypatterned heart disease. In TCM, the head and the upper thorax (上 焦, upper Jiao) are associated with the Heart. Heart plays a central role that any disorder affecting the Heart will also affect other organs, and vice versa. Cardiovascular disorders and organ-related specific symptoms attributed to the Heart are loss of memory, insomnia and psychosomatic problems

\section{Glance at Qi and Blood in TCM}

Yin-Yang balance, which distinguishes TCM from Western medicine, is the unique concept and one of the key principles of traditional Chinese medicine. The concept also serves as the foundation and the guideline for explaining etiology of diseases that are understood as a loss of balance between Yin and Yang as shown in Figure 1 [4]. Blood ( $\square$, Xue), classified into Yin and derived mainly from gu qi (款氣, food qi) of the food produced by the Spleen, is considered to be the nourishing agent in respect of TCM. The other source that generates Blood is Kidney since it stores Jing ( $\square$, essence) that can produce marrow. Marrow then generates bone marrow contributing to manufacturing Blood. On the other hand, Qi (氣), viewed as Yang and the moving agent to maintain the circulation in body, interacts with Blood [4]. Overall, the circulation of Qi and Blood in the body should be constant, or the pain shall occur when the "free flow" of Qi and Blood is disrupted [5]. In other 
words, the relationship between Qi and Blood can be interpreted to be inter-promoting, inter-dependent, and inter-transforming.

\begin{tabular}{|cc|}
\hline$\underline{\text { Yin }}$ & $\underline{\text { Yang }}$ \\
\hline & \\
Material & Function \\
Blood & $\mathrm{Qi}$ \\
Zang & $\mathrm{Fu}$ \\
Yin meridian & Yang meridian \\
\hline Figure 1: Characteristics of Yin-Yang. \\
\hline
\end{tabular}

The functions of Blood include moistening and nourishing the skin, muscles, sinews, bones and internal organs. The concept that Qi is "the commander of Blood" and Blood is "the mother of Qi" in TCM highlights Blood nourishes Qi and Qi moves Blood and suggests one is inconceivable without the other [6]. The circulation of Qi and Blood depends on the correct, inter-dependent functioning of ZangFu organs, involving in Lung, Heart, Liver, Spleen, Kidney, and San Jiao. One thing in common among three Zangs, Liver, Spleen, and Kidney, is closely connected with Blood as shown in Table 1. In the pathological perspective, the disease is caused by plaque building up along the inner walls of the arteries of the heart, which narrows the arteries down and reduces blood flow to the heart. These symptoms are viewed from the perspective of TCM as the patterns of Qi stagnation and Blood stasis, which are related to Liver. In the meantime, plaque is viewed as the phlegm derived from Dampness with the invasion of LV qi to Spleen.

Table 1: Functions of Zang organs related to Blood.

\begin{tabular}{|c|c|}
\hline Zang & Functions \\
\hline Liver & Stores Blood and regulates the volume of Blood \\
\hline Spleen & Controls Blood \\
\hline Kidney & Controls Bone \\
\hline
\end{tabular}

Table 2: Signs of Blood deficiency.

\begin{tabular}{|c|c|}
\hline Signs & Explanations in TCM \\
\hline $\begin{array}{c}\text { More palpitations pronounced } \\
\text { in the evening }\end{array}$ & $\begin{array}{c}\text { Heart Blood Deficiency affects Heart } \\
\text { Qi on account of that Blood is Yin. }\end{array}$ \\
\hline $\begin{array}{c}\text { Insomnia, poor memory, } \\
\text { anxiety, dream disturbed sleep, } \\
\text { easily startled }\end{array}$ & $\begin{array}{c}\text { Heart governs Blood and insufficient } \\
\text { Blood cannot nourish Brain, leading } \\
\text { to Heart unable to properly housing } \\
\text { the Shen. }\end{array}$ \\
\hline $\begin{array}{c}\text { Dull pale complexion, pale lips, } \\
\text { dizziness }\end{array}$ & Due to Blood Deficiency \\
\hline
\end{tabular}

The Liver qi ensures Qi to circulate consistently and smoothly in all parts of the body, regulating and connecting to Spleen and promoting the digestion functions to produce and hold Blood. External factors like Wind and Heat, emotional disturbance, bad diet, physical trauma, and improper exertion in activities, such as sex or excessive study and thinking result in the imbalance of Qi and Blood [4]. Pain is mainly a presentation of disturbances in the circulation of Qi and Blood, indicating the stagnation of Qi and stasis of Blood, or Qi deficiency and Blood deficiency. The organ that causes these is Heart because Heart dominates Blood and blood vessels, and is the root controller of Zang-Fu organs [5]. However, Liver and Spleen play the important roles that cannot be ignored in TCM when the etiology is Blood deficiency with the following signs in Table 2.

Angina is the common symptom in patients with coronary disease. There could be various causative factors leading to pain, but the main pathology is either due to blockage and obstruction resulted from Qi stagnation or deficiency of Blood. Malfunction and dysfunction of rotting and ripening of Stomach, which controls digestion, and transforming and transporting of Spleen, which produces and holds Blood, occur when these two organs are invaded by the stagnated Liver qi. The quality of Plasma concentration, which contributes to the risk of cardiovascular disease when the level rises, is to a certain extent interpreted in the perspective of TCM as phlegm in this study. In the Five Elements theory, phlegm results from Spleen qi deficiency when Liver qi invades Spleen. Blood deficiency results actually from hypofunction in Blood production, excessive Blood consumption of Essence and Blood with prolonged illness or over-exhaustion. The palpitations occur when Blood supply to the Heart is insufficient, including volume or flow of Blood.

\section{Considerations of Protocol}

An experienced TCM practitioner or acupuncturists can only use four skills for diagnosis to identify Patterns and finally give prescriptions, compared to Western medicine physicians who mostly rely on scientific instruments. Patterns, which distinguish TCM from the Western medicine, should be the key concern rather than diseases for TCM and acupuncture practitioners in treatment. In the Bible of TCM, Yellow Emperor's Inner Classic (Huang Di Nei Jing), it is stated that both Qi and Blood deficiency and Qi deficiency occur at 49 in females, and at 56 in males in the volume of Essential Questions. In other words, this notion reminds healthcare providers that Blood deficiency and Qi deficiency can be seen commonly in the senior people. Impeded Qi results in static Blood and finally leads to Blood deficiency. For better results, Blood mobilization and Qi regulation need treatment at the same time. On the other hand, it deserves attention that Blood deficiency may be seen as the source of dampness when it is closely linked to Spleen qi deficiency.

The most critical notion that "Feng ( $\square$, Wind), the beginner of the illness." discussed in Huang Di Nei Jing attracts attention because Wind is considered to be the major cause of illness with its pernicious influence. It also explains that Blood Xu (血虛, Blood deficiency) generates Wind inside the body, which is the cause of stroke and refers to pain, spasm and hypotension. Choosing Shustream to treat Zang organs; He-sea to treat Fu organs." has been the best principle for prescriptions presented in the volume of Sù Wèn 
(पD, Essential Questions) of Huang Di Nei Jing. For the purpose of Qi-Blood balance, LV 3 (太沖, Taichong) and ST 36 (पDC, Zusanli) are essential acupoints in the protocol as shown in Figure 2. Clinical experiences show it is beyond doubt that these two acupoints in the protocol function well to the expectations. ST36 is located on the Foot Yangming meridian, which is Yang, and LV3 is on Foot Jueyin meridian that belongs to Yin. On the other hand, these two acupoints are classified as Earth in the Five-Element, indicating they can nourish Spleen qi and Stomach qi to expel phlegm out of the body with the free flow of LV qi [7]. The symptoms and signs of Heart-Blood deficiency may include palpitations, insomnia, mild anxiety, poor memory, dizziness, and dull-pale complexion. HT 7 (神門, Shenmen) is added for insomnia, dizziness, and dull-pale complexion with the function to tonify Blood to Heart.

\begin{tabular}{|lc|}
\hline Oi & BloOd \\
LV 3 & ST36 \\
Yin meridian & Yang meridian \\
Shu-stream & Lower He-sea \\
\hline Figure 2: Characteristics of LV3 and ST36. \\
\hline
\end{tabular}

\section{Conclusion}

Acupuncture protocols cannot obtain therapeutic effects and good results without accurate pattern identification based on Y-n-Yang balance in treating functional heat problems as well as difficulty caused by disorders of the autonomic nervous system. The beginning stages of coronary heart disease without any advanced narrowing coronary arteries are well suited for acupuncture interventions. However, good results cannot be expected if key symptoms are not identified following TCM theories.

\section{References}

1. Baker IDI Heart and Diabetes Institute. Protein and coronary heart disease: the role of different protein sources.

2. Clinton Colmenares. Protein links heart disease, diabetes.

3. Schreiner PJ, Morrisett JD, Sharrett AR, Patsch W, Tyroler HA, et al. (1993) Lipoprotein(a) as a risk factor for preclinical atherosclerosis. Arterioscler Thromb 13(6): 826-833.

4. Maciocia G (1989) The foundations of Chinese Medicine. Library of Congress Cataloging in Publication Data, NY, USA.

5. Sun P (2011) Treatment of Pain with Chinese Herbs and Acupuncture. Churchill Livingstone, NY, USA.

6. Zhu B, Wang H (2010) Basic Theories of Traditional Chinese Medicine. People's military medical press, PA, USA.

7. Hong TZ (2017) Exploring a New Extra Point for Subacute Cough: a Case Report. J Complement Med Alt Healthcare 3(4): 555619.

\section{(c) (i) This work is licensed under Creative}

To Submit Your Article Click Here: Submit Article

DOI: 10.32474/OAJCAM.2018.01.000108

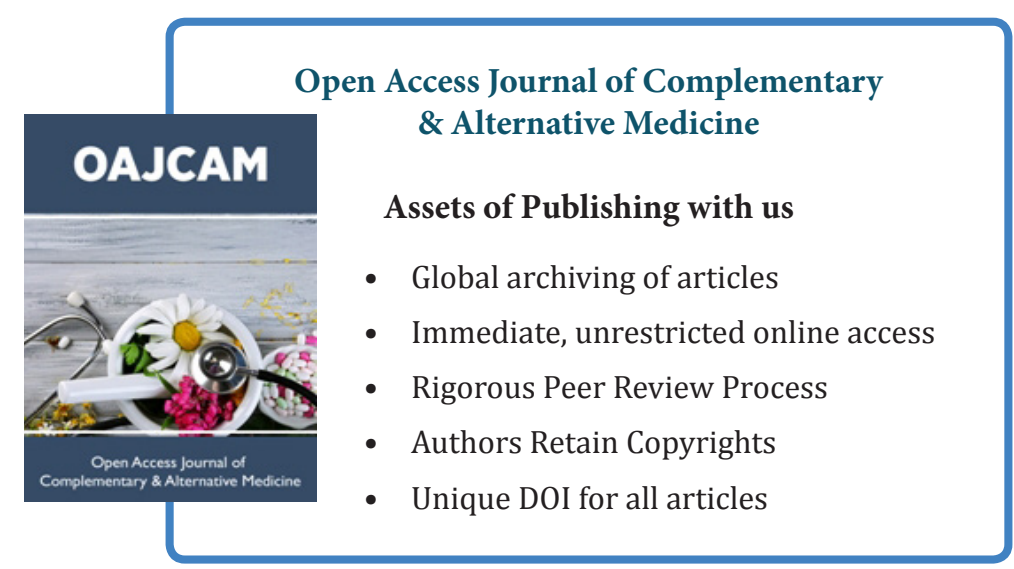

